\title{
Uric Acid: A Possible Mediator of the Adjuvant Effect of Alum in Mice Immunized with Ovalbumin
}

\author{
Nayla S. Al-Akl ${ }^{1}$, Marita Chakhtoura ${ }^{1}$, Natalie F. Kazzi ${ }^{1}$, Julnar Usta ${ }^{2}$, Camille A. Chamoun ${ }^{3}$, \\ Alexander M. Abdelnoor ${ }^{1}$ \\ ${ }^{1}$ Department of Microbiology and Immunology, Faculty of Medicine, American University of Beirut, Beirut, Lebanon; ${ }^{2}$ Department \\ of Biochemistry, Faculty of Medicine, American University of Beirut, Beirut, Lebanon; ${ }^{3}$ Lebanese Canadian Hospital, Beirut, Leba- \\ non. \\ Email: aanoor@aub.edu.lb
}

Received August $1^{\text {st }}, 2011$; revised October $4^{\text {th }}, 2011$; accepted October $13^{\text {th }}, 2011$.

\begin{abstract}
One proposed mechanism by which alum enhances an immune response is by its ability to induce an inflammatory response that results in the release of uric acid from necrotic cells. Uric acid is thought to be a mediator in enhancing the immune response. The aim of this study was to investigate the immunopotentiating effect of uric acid. Groups of BALB/c mice were injected intraperitoneally with ovalbumin, ovalbumin + alum, ovalbumin + uric acid, uric acid, alum, or allopurinol. Two other groups were pre-treated with allopurinol and were given ovalbumin + alum, or ovalbumin + uric acid 24 hours later. An additional two groups served as controls. On days 4, 7 and 10 post-injection, the numbers of Interleukin 4(IL-4) and Interferon- $\gamma(I F N-\gamma)$ secreting spleen cells were determined by the ELISPOT assay. Serum uric acid levels were determined using an autoanalyser and nitric oxide using the Greiss reagent. The groups that received alum + ovalbumin or uric acid + ovalbumin had the highest numbers of IL-4 and INF- $\gamma$ secreting cells as compared to all the groups. Allopurinol administration one day prior to alum + ovalbumin or uric acid + ovalbumin resulted in a decrease in the number of IL-4 and INF- $\gamma$ secreting cells when compared to alum + ovalbumin or uric acid + ovalbumin allopurinol - untreated groups. Groups that received alum, alum + ovalbumin, uric acid, and uric acid + ovalbumin had high serum uric acid levels as compared to all the groups. All groups that received alum had the highest levels of nitric oxide when compared to the groups that were not given alum. In conclusion, it appears that uric acid might be a mediator in the adjuvant effect of alum.
\end{abstract}

Keywords: ELISPOT, Interleukin-4, $\gamma$-Interferon, Nitric Oxide

\section{Introduction}

Alum is the most widely used adjuvant in human and veterinary vaccines $[1,2]$. Adsorption of antigen to alum is essential to enhance its immunogenicity [3]. Alum induces mainly a type- 2 immune response that promotes production of IL-4, IgE and IgG1 antibodies [1,4-7]. IL-4 secretion, caspase-1 activation, induction of certain chemokines, and complement activation by alum are all factors contributing to the type-2 immune response produced [1]. Several potential mechanisms are commonly cited to explain the method of action of this adjuvant: One proposed explanation is that alum can potentiate the immune response by retaining the antigen at the injection site suggesting a sustained release of antigen leading to the recruitment of Antigen Presenting Cells (APCs). This is known as the depot effect [3,8-10]. Another explanation is related to adsorption. Alum may help in converting the soluble antigen into a particulate multivalent form. Alum-antigen aggregates appear to be more immunogenic and can be efficiently phagocytosed by APCs such as macrophages and dendritic cells $[2,7,11,12]$. The ability of alum to cause inflammation at the site of injection is a third proposed mechanism [2]. Inflammation will result in tissue damage and necrosis at the injection site, and the necrotic cells release molecules that additionally activate dendritic cells [13]. These secreted molecules include uric acid (UA) crystals.

UA is a natural product of the purine catabolic path- 
way. It is known to be released from dying cells $[14,15]$. It is considered as an endogenous danger signaling molecule capable of stimulating the innate and adaptive immune systems $[16,17]$. UA crystals are capable of activating dendritic cells and inducing the Nalp3 inflammasome leading to activation of caspase- 1 and release of mature IL-1ß and IL-18 [18]. Crystals induce monocytes, macrophages, dendritic cells and epithelial cells to produce pro-inflammatory cytokines. These cytokines contribute to the immunopotentiating effect of UA [15]. The release of endogenous adjuvant would procure the immune system with enough stimulatory signals to respond to any antigen that is present and prevent tolerance. This process might elucidate the mechanism by which immune responses are generated to tumors and pathogens such as viruses or other antigens that require adjuvant support [19].

The activating effect of crystalline UA on the cells of the innate immune system leads ultimately to the activation of antigen specific T cells [15]. UA crystals will induce the expression of dendritic cell maturation markers CD80 and CD86 [19]. The mature dendritic cells then present the particulate antigen to the specific $\mathrm{T}$ cells and augment priming of $\mathrm{CD} 8+\mathrm{T}$ cells $[15,19]$. In addition, eliminating UA reduces the proliferation of ovalbuminspecific T cells [20]. However, others reported that UA can directly activate $\mathrm{T}$ cells in the absence of antigen presentation. When purified primary human $\mathrm{T}$ cells were incubated with UA, they became activated; as determined by the overexpression of T-cell activation markers such as CD25 by both CD4+ and CD8+ T cells. Additionally, the costimulatory molecule CD70 is over expressed on primary human CD4+ T cells treated with UA. CD70 is a surface molecule that is important in T cell-B cell stimulation [21].

UA increased IgG1 based humoral immunity when added with whole protein antigens or dying tumor cells, in mice [16]. In addition, mice that received a vaccine containing tumor antigens along with UA as an adjuvant had higher anti-tumor antibody levels than animals that received tumor antigens alone [22]. Hu et al. [14] detected the release of UA from tumor cells undergoing immune rejection and illustrated the fact that it had a significant role in the rejection process. Furthermore, the immune rejection process was substantially reduced by treatment with allopurinol and uricase, which markedly decreased the plasma concentrations of UA, indicating that UA is the molecule responsible for endogenous danger signaling [14].

In view of the fact that one of the potential explanations for the adjuvant effect of alum is through the induction of inflammation, which can result in UA release from necrotic cells; the aim of this study was to assess the role of UA in the adjuvant effect of alum.

\section{Materials and Methods}

\subsection{Reagents}

Alum (Allhydrogel, composed of Aluminum hydroxide Gel A; Brenntag Biosector, Denmark) was diluted with an equal volume of normal saline (Alfa Laboratories, Sehaileh, Lebanon). A $0.2 \mathrm{mg} / \mathrm{ml}$ solution of uric acid crystals ( $>99 \%$ purity; Sigma-Aldrich, St. Louis, MO, USA) in saline was used. A $1 \mathrm{mg} / \mathrm{ml}$ solution of Allopurinol (Sigma-Aldrich, St. Louis, MO, USA) was prepared in normal saline. Ovalbumin was prepared by removing the yolk and lyophilizing the remaining ovalbumin-containing egg white portion and a $0.8 \mathrm{mg} / \mathrm{ml}$ solution in saline was prepared. Alum-adsorbed ovalbumin was prepared by mixing equal volumes of $0.8 \mathrm{mg} / \mathrm{ml}$ ovalbumin and diluted alum.

\subsection{Mice}

Ninety female BALB/c mice, (6 - 8 wk old), were purchased from the Animal Care Facility at the American University of Beirut. They were divided into 10 groups of 9 mice each and received different intraperitoneal injections. The injection protocol that was followed is given in Table 1. All experiments were approved by the Institutional Animal Care and Use Committee (IACUC) at the Faculty of Medicine at the American University of Beirut.

\subsection{Procurement of Specimens}

On days 4, 7 and 10 post-injection, 3 mice from each group were anesthetized with a $0.5 \mathrm{ml}$ of a mixture of $0.12 \mathrm{ml}$ ketamine (final concentration $12 \mathrm{mg} / \mathrm{ml}$ ), $0.03 \mathrm{ml}$ xylazine (final concentration $1.2 \mathrm{mg} / \mathrm{ml}$ ), and $0.35 \mathrm{ml}$ sterile distilled water. Spleens were removed and pooled for preparation of a single cell suspension. Next the thoracic cavity was opened and blood was collected by cardiac puncture. Blood from each group was pooled; the serum was separated and used for uric acid and nitric oxide determinations.

\subsection{Preparation of Spleen Cell Suspensions}

Spleens were cut and grinded while adding RBC lysis solution (eBioscience, San Diego, CA). The resulting cells were suspended in RPMI culture medium (Gibco, Invitogen, Carlsbad, CA) + 1\% L-Glutamine (Irvine Scientific, Santa Ana) $+10 \%$ heat inactivated fetal calf serum (GIBCO, Invitrogen, Carlsbad, CA). Cells were centrifuged, resuspended in sterile freezing medium composed of RPMI $+1 \%$ Glutamine $+50 \%$ heat inactivated fetal calf serum $+10 \%$ dimethylsufoxide (DMSO; Sigma Chemicals co., MO, USA), then transferred to vials and stored in an isopropanol freezing container at $-80^{\circ} \mathrm{C}$ 
Table 1. Protocol used to investigate the effect of Alum and Uric Acid on the immune response.

\begin{tabular}{cccc}
\hline \multirow{2}{*}{ Group } & Group Description & \multicolumn{2}{c}{ Treatment } \\
\cline { 3 - 4 } & Negative Control & - & Day 0 \\
\hline 1 & Negative Control & - & No injection \\
2 & Positive Control & - & Sterile Saline (vehicle) \\
3 & Test Group & - & Ovalbumin $0.4 \mathrm{mg} / \mathrm{mouse}$ \\
4 & Test Group & Allopurinol $0.5 \mathrm{mg} / \mathrm{mouse}$ & Alum preparation \\
5 & Test Group & - & Alum + Ovalbumin $(0.4 \mathrm{mg}$ Ovalbumin $/ \mathrm{mouse})$ \\
6 & Test Group & - & Alum + Ovalbumin $(0.4 \mathrm{mg}$ Ovalbumin $/ \mathrm{mouse})$ \\
7 & Test Group & Uric acid $(0.1 \mathrm{mg} / \mathrm{mouse})$ \\
8 & Test Group & Allopurinol $0.5 \mathrm{mg} / \mathrm{mouse}$ & *Uric acid $(0.1 \mathrm{mg} / \mathrm{mouse})+$ Ovalbumin $(0.4 \mathrm{mg} / \mathrm{mouse})$ \\
9 & Test Group & - & Allopurinol $0.5 \mathrm{mg} / \mathrm{mouse}$ \\
\hline
\end{tabular}

overnight. The vials were then transferred to a liquid nitrogen-filled storage tank to maintain samples at a temperature below $-135^{\circ} \mathrm{C}$ for use in the ELISPOT technique.

\subsection{Enzyme-Linked Immunosorbent Spot (ELISPOT)}

The ELISPOT mouse Interferon- $\gamma$ (IFN $\gamma$ ) kit (Autoimmune Diagnostika GnBH, Germany) and the mouse Interleukin-4 (IL-4) ELISPOT plus kit (MABTECH AB, Sweden) were used. Procedures were performed according to the manufacturer's protocol and each sample was run in duplicate. For the ELISPOT procedure the frozen cells were thawed and viability was determined prior to loading them onto the ELISPOT plates.

\subsection{Serum Uric Acid Quantification}

The concentration of UA in serum was directly measured by an automated analyzer (Cobas Integra 400+, Roche, Basel, Switzerland).

\subsection{Serum Nitric Oxide (NO) Quantification}

The concentration of $\mathrm{NO}$ in the serum was indirectly measured using the Greiss reagent kit (Sigma Chemicals co., MO, USA). The procedure described by the manufacturer was followed.

\subsection{Statistical Analyses}

Statistical analyses were performed using non parametric tests. To test for correlation between continuous variables, Spearman's rho test was used. Results were considered significant at a $p$ value of 0.05 or less.

\section{Results}

\subsection{Interleukin-4 (IL-4)}

As shown in Figure 1, the number of IL-4 secreting cells was 4, 12 and 10 Spot forming units (SFU) on days 4, 7 and 10 respectively in the untreated controls and 13, 6 and 17 SFU respectively in the saline treated controls.

Immunization with ovalbumin, $184 \mathrm{SFU}, 97 \mathrm{SFU}$ and 80 SFU were obtained on days 4,7 and 10 post-immunization respectively. There was an increase in the number of IL-4 secreting cells when alum was given with ovalbumin compared to the numbers obtained in the group immunized with ovalbumin. Two hundred and sixty eight, 446 and 190 SFU on days 4,7 and 10 postimmunization respectively were obtained. These increases were diminished when mice were pretreated with allopurinol; 70, 66 and 78 SFU on days 4, 7 and 10, respectively. There was an increase in the number of IL-4 secreting cells when UA was given with ovalbumin on days 7 (208 SFU) and 10 (132 SFU) post-immunization compared to the numbers obtained in the group immunized with ovalbumin. These increases were diminished in mice pretreated with allopurinol; 78 and 32 SFU on days 7 and 10 respectively.

\subsection{Interferon- $\gamma($ IFN $\gamma)$}

Referring to Figure 2, the number of INF- $\gamma$ secreting cells was 28, 25 and 15 SFU on days 4,7 and 10 respectively for the untreated controls and 23, 25 and $30 \mathrm{SFU}$ respectively for the saline treated controls. Immunization with ovalbumin resulted in 35 SFU, 38 SFU and 38 SFU on days 4, 7 and 10 post-immunization respectively. 


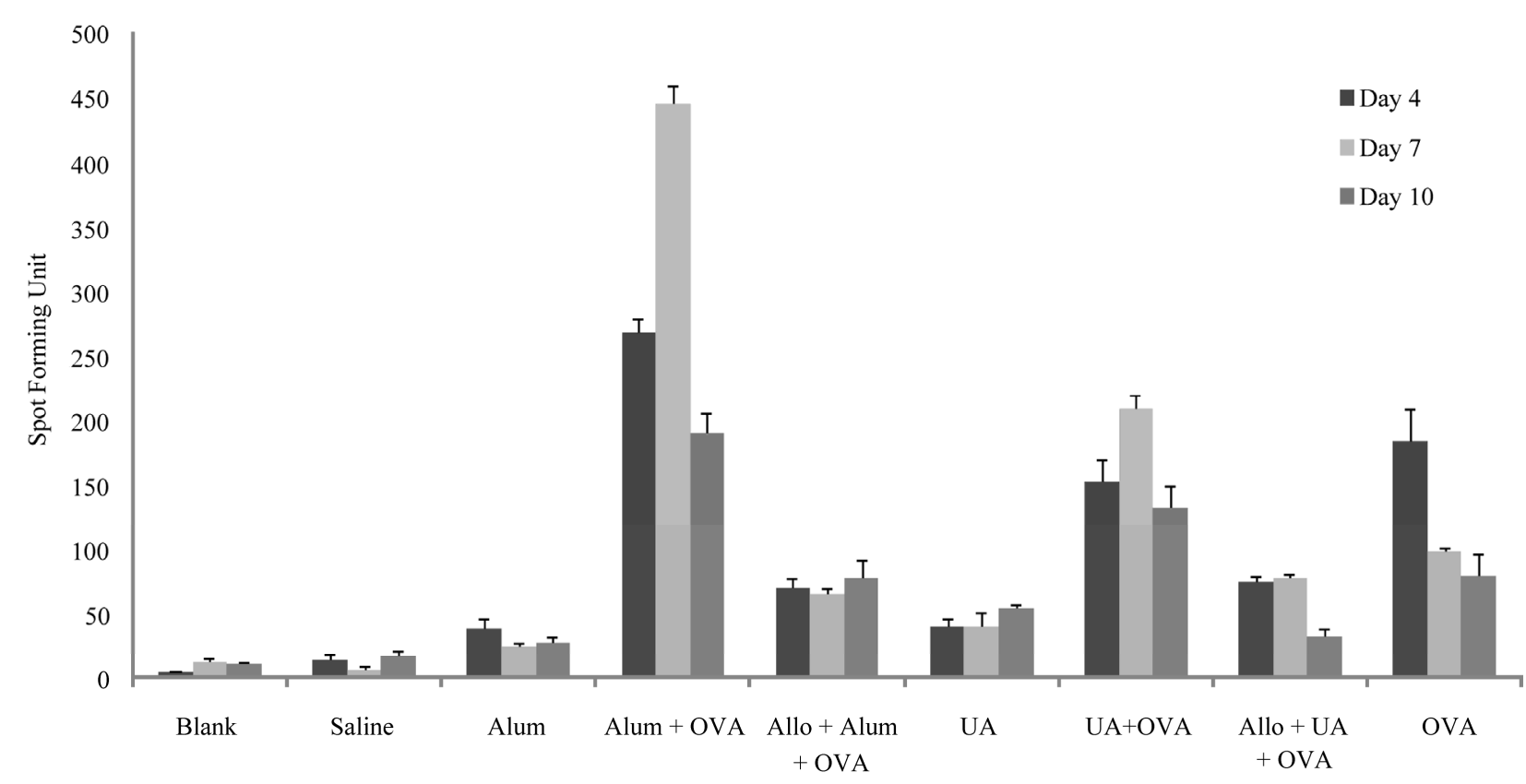

Figure 1. Number of cells actively secreting IL-4 in the different groups of mice on days 4, 7 and 10 post-stimulations as detected by the ELISPOT. Results show the average of two measurements for the number of spots forming units; standard deviations are also shown in the graph. Abbreviations are indicated as: Uric Acid (UA), Ovalbumin (OVA) and Allopurinol (Allo).

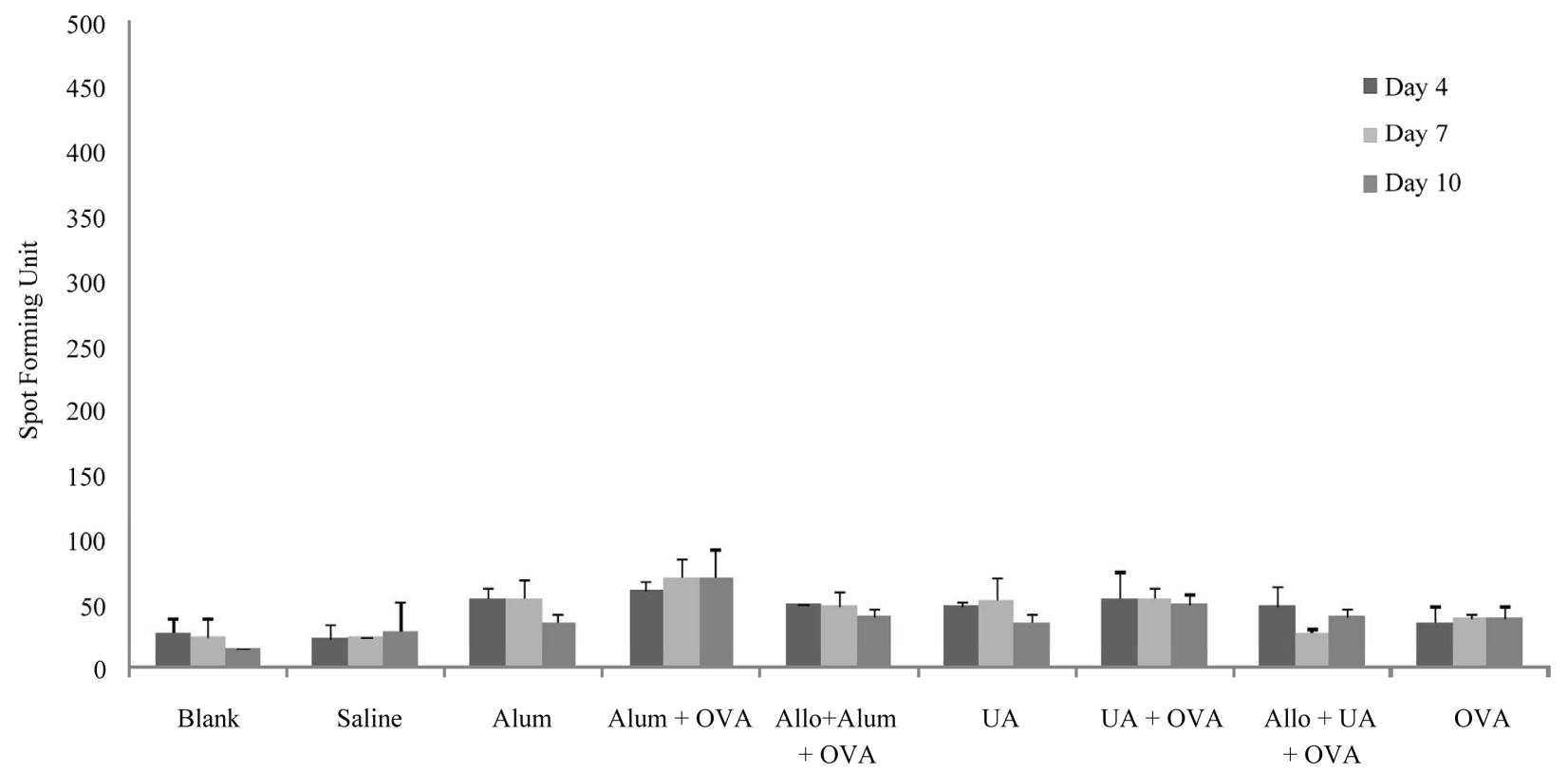

Figure 2. Number of cells actively secreting IFN $\gamma$ in the different groups of mice on days 4,7 and 10 post-stimulations as detected by the ELISPOT. Results show the average of two measurements for the number of spots forming units; standard deviations are also shown in the graph. Abbreviations are indicated as: Uric Acid (UA), Ovalbumin (OVA) and Allopurinol (Allo).

There was an increase in the number of IFN $\gamma$ secreting cells when alum was given with ovalbumin compared to the numbers obtained in the group immunized with ovalbumin alone. Sixty, 70, and 70 SFU on days 4, 7 and 10 post-immunization respectively were obtained. These increases were diminished in mice pretreated with al- 
lopurinol; 40, 48 and 40 SFU on days 4, 7 and 10, respectively. The number of IFN $\gamma$ secreting cells was increased when UA was given with ovalbumin on days 4 (55 SFU), 7 (55 SFU) and 10 (50 SFU) post-immunization compared to numbers obtained in the group immunized with ovalbumin. These increases were diminished in mice pretreated with allopurinol; 48, 27 and $40 \mathrm{SFU}$ on days 4, 7 and 10 respectively.

\subsection{Serum Uric Acid (UA) Levels}

UA levels are shown in Figure 3. Compared to the untreated and saline treated groups, UA levels increased in the groups given ovalbumin on days 4 and 7 post-injection ( 3 and $3.4 \mathrm{mg} / \mathrm{ml}$, respectively), ovalbumin + alum (4.3, 3.4 and $4.3 \mathrm{mg} / \mathrm{ml}$, on days 4,7 and 10 respectively), and $\mathrm{UA}+$ ovalbumin $(5.5,5.6$ and $5.1 \mathrm{mg} / \mathrm{ml}$, on days 4 , 7 and 10 respectively). Pretreatment with allopurinol had a minor effect on UA levels in the group given ovalbumin + alum $(4.2,3.4$ and $3.4 \mathrm{mg} / \mathrm{ml}$ on days 4,7 and 10 respectively). Pretreatment with allopurinol diminished the UA levels in the group given UA + ovalbumin (3.4, 0.7 and $3.5 \mathrm{mg} / \mathrm{ml}$ on days 4,7 and 10 respectively).

\subsection{Serum Nitric Oxide (NO) Levels}

NO levels are given in Figure 4. NO levels in the group that was immunized with ovalbumin was not significantly different from the control groups; 209, 203 and $180 \mu \mathrm{M}$ at 4,7 and 10 days post-immunization, respectively. NO levels in the group that received alum + ovalbumin were 285,420 and $318 \mu \mathrm{M}$ at 4,7 and 10 days post-immunization, respectively and were higher than the group that received ovalbumin. There was no significant difference in NO levels between the group that received
$\mathrm{UA}+$ ovalbumin $(181,212$ and $200 \mu \mathrm{M}$ at 4,7 and 10 days post-immunization, respectively) and the group that received ovalbumin.

\subsection{Correlation between Results}

IL-4 secretion from cells and UA serum levels of the different groups were compared to the control groups. Statistical results showed a positive linear correlation between these two variables for all the groups on all the days, indicating that whenever uric acid level increased, IL-4 secretion increased as well. However, the correlation was significant only for the groups that received ovalbumin, alum, alum + ovalbumin, and the allopurinol pretreated group that received alum + ovalbumin as compared to the control groups on day 4 with a spearman's rho of 0.829 and a $p$ value of 0.042 .

IFN $\gamma$ secretion and UA levels between the different groups and the control groups were compared. All groups showed a positive linear correlation between the two variables on all the days, indicating that when UA levels increased an elevation of IFN $\gamma$ secretion from cells was observed. On day 4, all groups expressed a very strong significant correlation when compared to the control groups. Ovalbumin, alum, alum + ovalbumin, and the pretreated allopurinol that received alum + ovalbumin groups as compared to the control groups resulted with a spearman's rho of 0.886 and a $p$ value of 0.019 . Groups that received UA, UA + albumin, allopurinol, and the allopurinol pretreated group that received UA + ovalbumin, as compared to the control groups, showed a spearman's rho of 0.901 and $p$ value of 0.006 .

However, on days 7 and 10 though a correlation existed between all the groups and the control groups, it

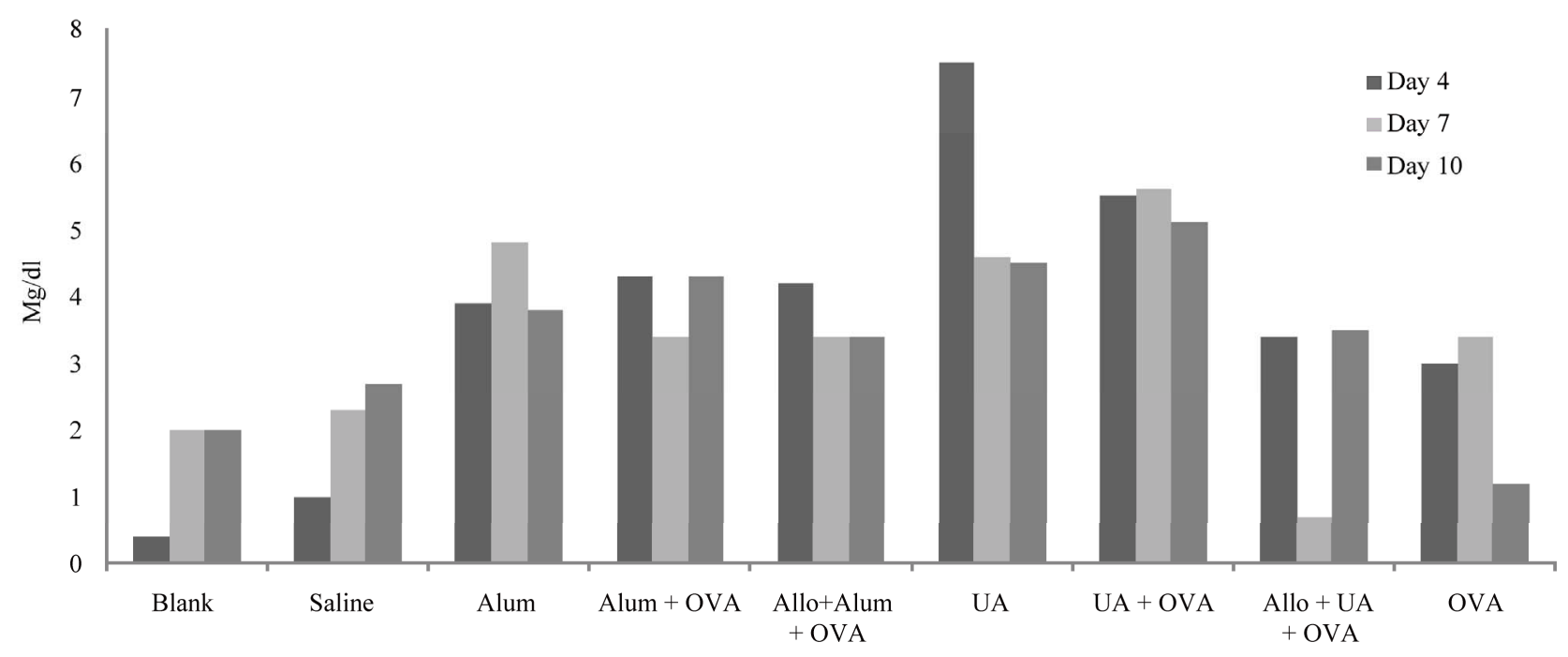

Figure 3. Serum uric acid levels in different groups of mice on days 4, 7 and 10 post-stimulation. Abbreviations are indicated as: Uric Acid (UA), Ovalbumin (OVA) and Allopurinol (Allo). 


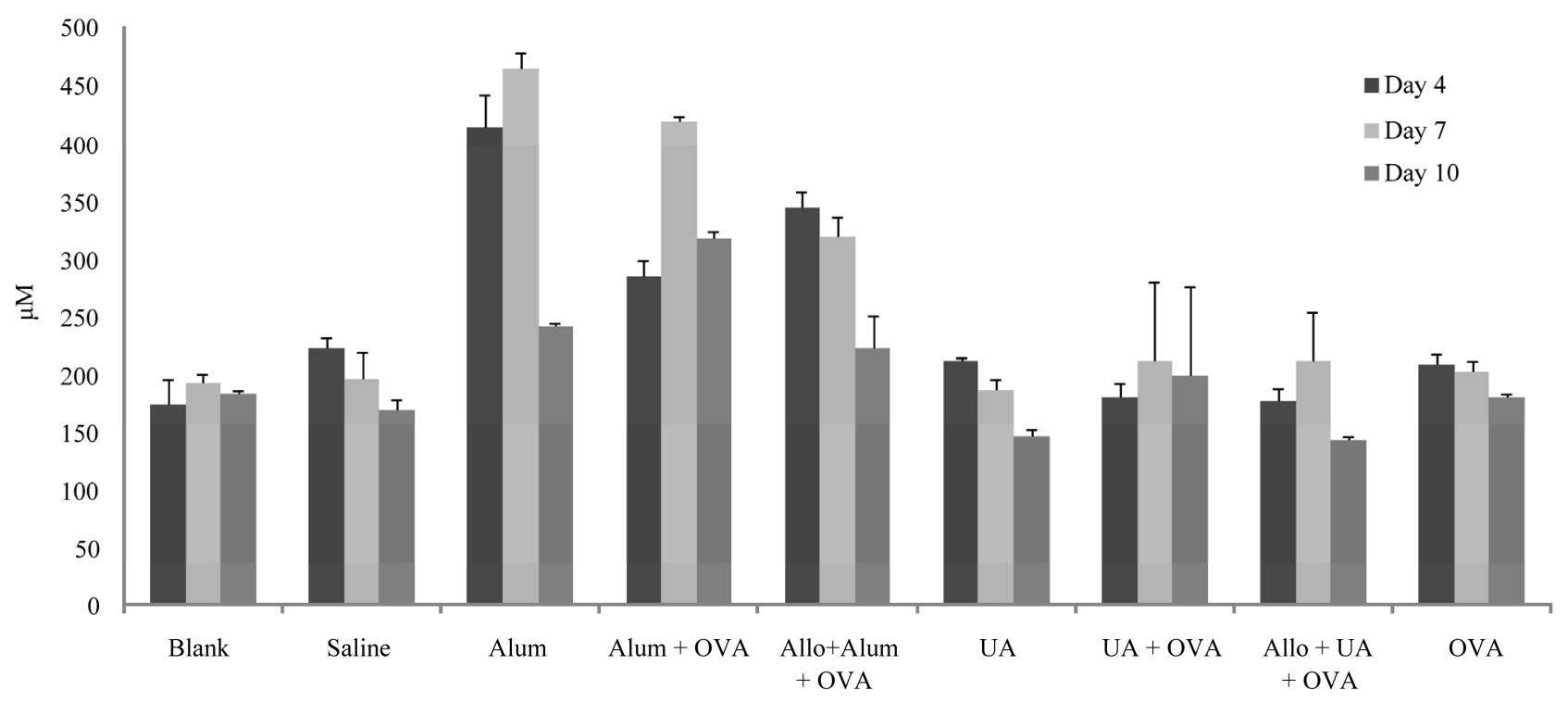

Figure 4. Results of serum nitric oxide levels in different groups of mice on days 4, 7 and 10 post-stimulation. The average of duplicate run for each sample is shown along with the standard deviations. Abbreviations are indicated as: Uric Acid (UA), Ovalbumin (OVA) and Allopurinol (Allo).

was only significant in the groups that received ovalbumin, alum, alum + ovalbumin, and the group pretreated with allopurinol and given alum + ovalbumin as compared to the control groups on day 7 , where a borderline significant correlation with a spearman's rho equal to 0.801 and $p$ value equal to 0.056 was obtained.

\section{Discussion}

Alum is an adjuvant that has been approved by the United States Food and Drug Administration (FDA) for human use. Its mechanism of action is not fully understood. Recently it has been proposed that alum induces inflammation at the site of injection leading to the liberation of UA which contributes to adjuvanticity. The aim of this study was to investigate the adjuvant effect of UA in mice. This was done by determining the number of IL-4 and IFN $\gamma$ secreting spleen cells following the administration of different preparations listed in Table 1. It is worth noting that IL-4 is a T-helper-2 (Th2) cytokine and IFN $\gamma$ is a T-helper-1 (Th1) cytokine.

After intraperitoneal administration of the different preparations into mice, the highest numbers of cytokinesecreting spleen cells were observed on day 7 , in most of the groups, in particular the groups that received alum + ovalbumin. This observation concurs with the report of Kool et al. [2], who showed that peak levels for the recruitment of immune cells to the spleen, appears mainly by day 7 after immunostimulation through the intraperitoneal route.

When ovalbumin was injected alone, it caused a significant increase in the number of IL-4-secreting spleen cells. There was a further significant increase in the number of these cells in the group of mice that received alum + ovalbumin. These results concur with the well established fact that alum when injected with an antigen is a potent adjuvant that induces a Th2 response leading to the production of IL-4 [1,4-7].

Likewise, there were significant increases in IFN $\gamma$-secreting spleen cells in most of the test groups, as compared to the group that received ovalbumin alone. However, increases were less than that obtained for the IL-4-secreting spleen cells. This could be attributed to the fact that IL-4 when produced, suppresses the T-helper 1 response and thus decreases levels of IFN $\gamma$ produced [23].

The half life of UA in plasma has been reported to be $10.8 \mathrm{hrs}$ [24]. However, a baseline is maintained because UA that is eliminated is replaced by newly formed UA. Increased levels of UA are seen in certain diseases such as Gout. Since the mice used in this study were healthy, exogenous UA was given to one group to observe the effect of elevated levels of UA on the number of IL-4 and IFN $\gamma$ secreting cells.

It has been reported that UA has the capacity to induce a T-helper 2 response [16,22]. The increase in the number of IL-4-secreting spleen cells obtained when UA was used as an adjuvant is in support of these reports. Hence, our data support others who claim that UA is an endogenous adjuvant $[15,19]$. An increase in IFN $\gamma$ secreting cells was also observed in the group of mice that received UA + ovalbumin. This result suggests that UA can also potentiate a Th1 response. 
To determine whether alum caused the release of UA which in turn caused an increase in both IL-4- and IFN $\gamma$ secreting spleen cells, mice were pre-treated with allopurinol, an inhibitor of UA production [25,26]. Pretreatment with allopurinol resulted in a decrease in the number of both IL-4- and IFN $\gamma$-secreting spleen cells in mice given alum + ovalbumin or UA + ovalbumin. If IL-4 production is to be equated to a Th2 response and IFN $\gamma$ to a Th1 response then it appears that one possible mechanism by which alum exerts its adjuvant effect is by liberating UA from cells which in turn promotes Th2 and to a lesser extent Th1 responses.

In an attempt to demonstrate whether alum's immunostimulatory effect might be through the induction of the endogenous danger signal UA, serum UA levels were measured for all the groups. As expected, groups that received UA or alum injections had high levels of serum UA that positively correlated with the increased numbers of IL-4 and IFN $\gamma$ secreting cells. This indicates that whenever UA levels increased IL-4 and IFN $\gamma$ secretion from spleen cells increased. Allopurinol successfully inhibited UA production as shown by the groups that received allopurinol prior to UA and ovalbumin injections.

Elevated NO levels can be considered an indicator of an inflammatory process [27]. There were elevated levels of NO in groups of mice that received alum. This result is supportive of the fact that alum is an inflammatory agent $[1,2]$. On the other hand, NO levels in mice that received UA were not significantly different than levels in controls. This could indicate that although UA is a product of alum-induced inflammation its adjuvant effect is inflammation-independent.

In conclusion, the fact that both alum and UA were shown to cause an increase in IL-4 and to a lesser extent IFN $\gamma$ secreting cells, and these increases were suppressed by allopurinol, it can be suggested that UA could be an intermediate in the pathway leading to the enhancement of an immune response by alum.

\section{REFERENCES}

[1] H. HogenEsch, "Mechanisms of Stimulation of the Immune Response by Aluminum Adjuvants," Vaccine, Vol. 20, Suppl. 3, 2002, pp. 34-39. doi:10.1016/S0264-410X(02)00169-X

[2] M. Kool, T. Soullié ,M. Van Nimwegen, M. A. Willart, F. Muskens, S. Jung, H. C. Hoogsteden, H. Hammad and B. Lambrecht, "Alum Adjuvants Boosts Adaptive Immunity by Inducing Uric Acid and Activating Inflammatory Dendritic Cells," The Journal of Experimental Medicine, Vol. 205, No. 4, 2008, pp. 869-882. doi:10.1084/jem.20071087

[3] E. B. Lindblad, "Aluminum Adjuvants in Retrospect and
Prospect," Vaccine, Vol. 13, No. 22, 2004, pp. 3658-3668. doi:10.1016/j.vaccine.2004.03.032

[4] E. De Gregorio, E. Tritto and R. Rappuoli, "Alum Adjuvanticity: Unraveling a Century Old Mystery," European Journal of Immunology, Vol. 38, No. 8, 2008 pp. 20682071. doi:10.1002/eji.200838648

[5] J. N. Francis and S. R. Durham, "Adjuvants for Allergen Immunotherapy: Experimental Results and Clinical Perspectives," Current Opinion in Allergy and Clinical Immunology, Vol. 4, No. 6, 2004, pp. 543-548. doi:10.1097/00130832-200412000-00012

[6] M. B. Jordan, D. M. Mills, J. Kappler, P. Marrack and J. C. Cambier, "Promotion of B Cell Immune Responses via an Alum-Induced Myeloid Cell Population," Science, Vol. 304, No. 5678, 2004, pp. 1808-1810. doi:10.1126/science.1089926

[7] H. Li, S. Nookala and F. Re, "Aluminum Hydroxide Adjuvants Activate Caspase-1 and Induce IL-1 Beta and IL-18 Release," Journal of Immunology, Vol. 178, No. 8, 2001, pp. 5271-5276.

[8] J. C. Cox and A. R. Coulter, "Adjuvants-A Classification and Review of Their Modes of Action," Vaccine, Vol. 15, No. 3, 1997, pp. 248-256. doi:10.1016/S0264-410X(96)00183-1

[9] M. Ulanova, A. Tarkowski, M. Hahn-Zoric and L. Hanson, "The Common Vaccine Adjuvant Aluminum Hydroxide Up-Regulates Accessory Properties of Human Monocytes via an Interleukin-4-Dependent Mechanism," Infection and Immunity, Vol. 69, No. 2, 2001, pp. 11511159. doi:10.1128/IAI.69.2.1151-1159.2001

[10] R. G. White, A. H. Coons and J. M. Connoly, "Studies on Antibody Production. III. The Alum Granuloma," Journal of Experimental Medicine, Vol. 102, No. 1, 1955, pp. 7382. doi:10.1084/jem.102.1.73

[11] G. L. Morefield, A. Sokolovska, D. Jiang, H. HegenESch, J. P. Robison and S. L. Hem, "Role of Aluminum-Containing Adjuvants in Antigen Internalization by Dendritic Cells in Vitro," Vaccine, Vol. 23, No. 13, 2005, pp. 15881595. doi:10.1016/j.vaccine.2004.07.050

[12] E. Tritto, F. Mosca and E. De Gregorio, "Mechanism of Action of Licensed Vaccine Adjuvants," Vaccine, Vol. 27, No. 25-26, 2009, pp. 3331-3334. doi:10.1016/j.vaccine.2009.01.084

[13] S. Galluci, M. Lolkema and P. Matzinger, "Natural Adjuvants: Endogenous Activators of Dendritic Cells," $\mathrm{Na}$ ture Medicine, Vol. 5, No. 11, 2000, pp. 1249-1255. doi: $10.1038 / 15200$

[14] D. E. Hu, A. Moore, L. Thomsen and K. Brindle, "Uric Acid Promotes Tumor Immune Rejection," Cancer Research, Vol. 64, No. 15, 2004, pp. 5059-5062. doi:10.1158/0008-5472.CAN-04-1586

[15] Y. Shi, J. E. Evans and K. L. Rock, "Molecular Identification of a Danger Signal That Alerts the Immune System to Dying Cells," Nature, Vol. 425, No. 6957, 2003, pp. 516-521. doi:10.1038/nature01991

[16] M. Behrens, W. Wagner, C. Krco, C. Erskine, K. Kalli, J. Krempski, E. Gad, M. Disis and K. Knutson, "The En- 
dogenous Danger Signal, Crystalline Uric Acid, Signals for Enhanced Antibody Immunity," Blood, Vol. 111, No. 3, 2008, pp. 1472-1479. doi:10.1182/blood-2007-10-117184

[17] R. L. Hunter, "Overview of Vaccine Adjuvants: Present and Future," Vaccine, Vol. 20, No. 3, 2002, pp. 7-12. doi:10.1016/S0264-410X(02)00164-0

[18] F. Martinon, V. Petrilli, A. Mayor, A. Tardivel and J. Tschopp, "Gout Associated Uric Acid Crystals Activate the NALP3 Inflammasome," Nature, Vol. 440, No. 7081, 2006, pp. 237-241. doi:10.1038/nature04516

[19] Y. Shi, W. Zheng and K. L. Rock, "Cell Injury Releases Endogenous Adjuvants That Stimulate Cytotoxic T Cell Responses," Proceedings of the National Academy of Science, Vol. 97, No. 26, 2000, pp. 14590-14595. doi:10.1073/pnas.260497597

[20] Y. Shi, S. A. Galusha and K. L. Rock, "Cutting Edge: Elimination of an Endogenous Adjuvant Reduces the Activation of CD8 T Lymphocytes to Transplanted Cells and in an Autoimmune Diabetes Model," Journal of Immunology, Vol. 176, No. 7, 2006, pp. 3905-3908.

[21] R. Webb, M. Jeffries and A. H. Sawalha, "Uric Acid Directly Promotes Human T-Cell Activation," The American Journal of the Medical Sciences, Vol. 337, No. 1, 2009, pp. 23-27. doi:10.1097/MAJ.0b013e31817727af

[22] G. Vielhauer, E. Gad, W. Wagner, M. L. Disis and K. L.
Knutson, "The Endogenous Danger Signaling Molecule, Uric Acid, Converts Immunity from Non-Protective to Protective When Used as a Vaccine Adjuvant," American Association for Cancer Research, Vol. 46, 2005, Abstract 5167.

[23] J. M. Brewer, M. Conacher, A. Satoskar, H. Bluethmann and J. Alexander, "In Interleukin-4 Deficient Mice, Alum Not Only Generates T Helper-1 Responses Equivalent to Freund's Complete Adjuvant, But Continues to Induce T Helper-2 Cytokine Production," Journal of Immunology, Vol. 26, No. 9, 1996, pp. 2062-2066.

[24] W. S. Waring, J. D. Webb and R. J. S. Maxwell, "Systemic Uric Acid Administration Increases Serum Antioxidant Capacity in Healthy Volunteers," Journal of Cardiovascular Pharmacology, Vol. 38, No. 3, 2001, pp. 365-371. doi:10.1097/00005344-200109000-00005

[25] G. B. Elion, "The Purine Path to Chemotherapy," Bioscience Reports, Vol. 9, No. 5, 1989, pp. 509-529. doi:10.1007/BF01119794

[26] G. B. Elion, "Enzymatic and Metabolic Studies with Allopurinol," Annals of the Rheumatic Diseases, Vol. 25, No. 6, 1966, pp. 608-614.

[27] J. R. Parratt, "Nitric Oxide in Sepsis and Endotoxaemia," Journal of Antimicrobial Chemotherapy, Vol. 41, Suppl. A, 1998, pp. 31-39. doi:10.1093/jac/41.suppl 1.31 